the honours or postgraduate levels it also teaches entomology, parasitology and marine biology. If there is any special emphasis in the equipment provided, it is in the direction of ensuring that adequate arrangoments exist for teaching and research in the field of comparative physiology, because the future of zoology is so much bound up with this that no department can expect to contribute substantially to the progress of the subject unless it is well equipped
for such work.

The various rooms in the Department (about sixty) are so arranged that the circulation of large numbers of students is confined in the main to the two lowor floors, with the result that senior students and staff have the two upper floors to themselves. Thus the large locture theatre opens from the basement, and the main students' cloakrooms are on the same level. On the floor above is the large laboratory, with the zoology lecture room opening from the same passage. The floor next above this one is laid out primarily for senior physiological teaching and research, and also has a technicians' common room, a larger common room for the academic staff, and the library. The top floor provides private rooms for the staff and for postgraduate research, as well as the honours laboratory with an adjacent room for section-cutting.

Several other principles have been kept in mind during the planning. Plenty of store-rooms have been provided, and also an adequate number of photographic dark-rooms situated on different floors, so that technicians, research students and academic staff do not need to use the same ones. Further, the teaching laboratories have been supplied with large and well-equipped preparation rooms opening into them, and this much facilitates the handling of teiaching material. In connexion with the physiological section of the Department there is an aquarium in which various types of work can be done, and this room has a circulation of sea water through six small tank; and a circulation of fresh water through six others. In the basement there is a museum, and an innovation on the same floor. is a visitors' research laboratory in which are four cubicles, each of which can be used by a visiting scientist, as well as general facilities which visitors can share. There are also two constant-temperature rooms : and compressed air is laid on throughout the building. A laboratory properly equipped for the use of radioactive materials has been provided on the roof.

The whole planning of the internal detail of the Zoology Department was done, in consultation with the architects and the staff, by Prof. and Mrs. T. A. Stephenson.

Aberystwyth is in many ways very favourably situated for biological studies, since there are close at hand a wide variety of habitats, which include not only the sea and shore for marine studies, but also inland rivers and lakes, woodlands, bogs, moorland, sand-dunes and salt-marsh. Moreover, there is a large botany garden very close to the new building. In the past the Biological Departments wore handicapped by inadequate laboratory accommodation ; but with the large new laboratories and the close proximity of a wide variety of natural vegetation and fauna, the College can now offer opportunities for biological teaching and research which compare favourably with those provided elsewhere in the United Kingdom.

\section{T. A. STEPHENSON}

P. F. WAREINC:

\title{
BOUNDARY-LAYER RESEARCH IN FLUIDS
}

$\mathrm{W}$ HEN a body of streamlined shape moves through a fluid, the latter is disturbed, but in the main it is not carried along with the body. On the surface of the body, however, the fluid shares the body's motion, because viscosity prevents any slip occurring. The change in speed between the fluid at the surface adhering to it and the fluid in the external flow takes place in a thin layer, called the boundary layer. Boundary-layer flow has been intensively studied because many important practical effects depend on the nature of this flow. The fluid exerts a frictional retarding force on the body. This drag depends on the way in which the fluid speed varies across the boundary layer, and it tends to decrease as the boundary-layer thickness increases. It is increased if the flow in the boundary layer, instead of being completely smooth or laminar, is turbulent, with a small-scale eddying motion superimposed on the mean motion. Much work has therefore been devoted to the conditions which provoke the transition from laminar to turbulent flow in the boundary layer.

Another important practical problem is boundarylayer separation. Relative to the body, the boundary layer fluid has less momentum than that in the external flow. Hence if over the surface of the body the pressure rises in the stream direction, the bound. ary-layer fluid may be brought to rest, because it will tend to be retarded relatively more by the pressure gradients than the fluid in the external flow. A piling-up of low-speed fluid near the surface may therefore occur, which may make the streamlines in the external flow depart markedly from the shape of the body. A low-pressure region over the rear end of the body sometimes results, with a consequent large pressure-drag. Here again turbulent boundary layers behave very differently from laminar ones, being much less prone to separation. In recent years, with the advent of high-speed aircraft and missiles, aerodynamic heating has become of great importance. This is largely a boundary-layer effect, because it is mainly due to the kinetic energy of the free-stream air being converted into heat when, relative to the body, the air is slowed down by friction inside the boundary layer. Associated with their greater frictional drag, turbulent boundary layers tend to produce greater rates of heating than laminar ones.

It can be understood from the foregoing that the study of boundary layers as a special department of fluid mechanics has itself become specialized into several sub-departments. These specialist interests are exemplified by the papers presented at the meeting on "Boundary Layer Research" arranged by Dr. W. P. Jones of the Aerodynamies Division, National Physical Laboratory, on behalf of the N.A.T.O. Advisory Group for Aeronautical Research and Development and held in London during April 25-29. The topics discussed were grouped under the following headings: (1) effects of roughness on boundary-layer stability; (2) boundary-layer con- 
trol ; (3) stability of shear layers; (4) mechanism of transition and boundary-layer stability ; (5) threedimensional boundary layers ; $(6)$ turbulent boundary layers ; (7) heat transfer.

About forty papers were read by authors from Belgium, France, Germany, Great Britain, Italy, and the United States, and it is obviously out of place to attempt to summarize them all here. It may, however, be profitable to discuss the topics of some of the above headings in broad terms, in the light of the papers presented at the meeting.

The position at which transition to turbulent flow occurs on a smooth surface depends on several factors. If the surface is a flat plate parallel to the flow, it might at first sight be expected that the only factors affecting the distance $x_{t}$ of the transition position from the leading edge of the plate would be the density o, the viscosity $\mu$, and the velocity $V$ of the oncoming flow. Then $p V x_{t} / \mu$, which is a dimensionless quantity of the kind known as a Reynolds number, would take a definite value at transition. However, in practice the Reynolds number of transition is not uniquely defined. It is very dependent on the magnitude of any small disturbances present in the stream or on the nature of any imperfections in the surface. Small wave-like disturbances introduced into the laminar boundary layer by these means can become amplified and distorted until eventually the flow becomes turbulent. These processes have been for many years and still are the subjects of theoretical and experimental investigations, and many papers at the meeting were concerned with them. The initial small wave-like motions can be treated with some success mathematically because the problem can be linearized. The disturbance velocities are superimposed on those of the undisturbed flow, and any products of the disturbance terms in the equations are neglected, the first-order terms only being retained. A further simplification usually introduced is to consider only two-dimensional disturbances, such that the disturbance velocities do not vary along lines perpendicular to the flow across the flat plate on which the boundary layer is formed. The justification for this is that it can be shown that such two-dimensional disturbances are more rapidly amplified than three-dimensional ones. However, three-dimensional disturbances that are not too oblique can be amplified, and this process has recently been studied because experiment has emphasized that the final process of breakdown into turbulent flow is essentially three-dimensional, with disturbance velocities in all directions superimposed on the mean flow. However, the disturbances considered by linear theory, even in its three-dimensional form, are different in character from those which immediately precede the breakdown into turbulent flow, and to help to bridge this gap in our theoretical understanding, other workers have taken into account some of the higher-order non-linear terms in the equations. Another difficult problem has been the extension of the linear small-disturbance theory to high-speed flow. It has recently been discovered that the earlier version of this extension is in some respects unsound, and a more adequate theory is now being developed.

Surfaces such as aircraft wings are never perfectly smooth, and it is therefore important to investigate what effects randomly distributed elements of roughness are likely to have on transition to turbulent flow. This problem is too complicated to study theoretically, and its experimental investigation is not easy, because it is complicated by the large number of factors defining the roughness - the spacing, height, and shape of the excrescences may all vary. For a band of roughness elements of given geometry situated transversely to the flow, transition will be provoked immediately downstream of the band if the roughness size exceeds a certain minimum. This critical size can be defined by a Reynolds number based on the height of the excrescences and on the velocity, density, and viscosity in the oncoming boundary layer at a distance from the wall equal to the height of the excrescences. There is some evidence that such a critical Reynolds number tends to be constant irrespective of the free-stream Mach number (the ratio of the speed to the local speed of sound, and also irrespective of heat transfer between the stream and the surface. If this is so, cooling a surface with distributed roughness would tend to provoke earlier transition, since it would increase the roughness-element Reynolds numbers. This is in contrast with the behaviour of boundary layers on smooth surfaces, where cooling delays transition.

Results of wind-tunnel investigations of transition rnay not be in all respects valid for free-flight conditions. Turbulence in the free stream outside the boundary layers may be much higher in a wind tunnel than in flight, where small-scale turbulence is usually negligible. In low-speed wind tunnels this freestream turbulence may be reduced to a low level by the provision of a large settling chamber containing gauze screens through which the air is made to pass upstream of the working section, where the model under test is situated. However, in high-speed wind tunnels the free-stream turbulence cannot be so readily got rid of, since in the main it comes from acoustic radiation from the eddying motions in the turbulent boundary layers on the tunnel walls. A tentative partial explanation of why this radiation is greater at supersonic than at subsonic speeds is as follows. Eddies in the boundary layer move along with the local speed, so that the external flow moves past them. In effect, from the point of view of the external flow an eddy may form a moving local excrescence or depression. When the speed of the relative motion between the external flow and the eddy is less than that of sound, the disturbance generated in the external flow would be expected to be diffuse, tending perhaps to cancel with contributions from other eddies. On the other hand, when the speed of the relative motion exceeds that of sound, the effects of the disturbances are presumably concentrated along shock waves, and they might tend to persist without cancellation in the external flow. If this explanation is correct, it implies that the bulk of the radiated energy comes from the eddies in the inner part of the boundary layer, moving at supersonic speeds relative to the external flow, and not from eddies in the outer part of the boundary layer, where the relative motion is subsonic. In any event, the radiated energy is associated somehow with the turbulent motions in the boundary layer. If the tunnel could be run at sufficiently low pressures it would be possible to have laminar boundary layers on the tunnel walls, which would greatly reduce the free-stream turbulence-level. Under these conditions, however, the tunnel could not readily be used to study transition, because the boundary layers on any model in the tunnel would normally be entirely laminar.

It is still a matter for conjecture as to how similar turbulent boundary layers in supersonic flow are to 
those in low-speed flow. At high speeds, but not at low speeds, significant temperature and density fluctuations occur, and at first sight it would appear that they must completely alter the nature of the flow. However, it can be argued that the velocity fluctuations are to some extent independent of the thermal effects, and many of the overall characteristics of supersonic turbulent boundary layers are sufficiently similar to the corresponding ones in lowspeed flow to encourage the view that there is a fundamental similarity in the underlying processes of energy exchange between the mean motion and the turbulent oddies, at least up to moderate supersonic speeds. The energy radiated to the free stream, discussed above, may not play a very big part in the overall energy balance.

A disturbing feature that has been noticed in tests of supposedly two-dimensional turbulent boundary layers is the presence of three-dimensional effects Such characteristics as the boundary-layer thickness have been found to be non-uniform for the boundary layer on a flat plate along lines across the plate perpendicular to the flow. These effects are not to be traced to any small imperfections in the leading edge of the plate, but they seem to depend on the condition fo the screens in the settling chamber of the tunnel. It has been shown that characteristics such as the frictional drag, as calculated from the rate of growth of the boundary layer, are considerably affected by taking into account the spanwise variations.

Spanwise variations of a somewhat similar kind have been observed in regions where a laminar boundary layer re-attaches itself to a surface downstream of a region of separation. Liquid which, after being smeared on to the surface, evaporates most rapidly from regions where the skin friction is highest, reveals a streaky pattern with streaks parallel to the flow.
This is suggestive of a number of parallel vortices in the boundary layer, with their axes in the direction of the stream. Such vortices are formed when a fluid flows over a concave surface, as has been known for a long time. The laminar boundary layer on such a surface is unstable because if a relatively fast-moving particle of fluid gets displaced nearer to the surface, where the general speed of the fluid is less, it comes into a region where its centrifugal force exceeds the restoring force exerted by the local pressure field, which is such as to balance the local, smaller, centrifugal forces. Thus the motion is unstable, and pairs of counter-rotating vortices result. It would seem at first sight reasonable to expect a similar instability in a re-uttachment region, where the streamlines tend to be concave even when the solid surface is flat. However, it was found that the streaky pattern persisted even when the surface on which the re-attachment occurred was made convex. This would presumably damp out any instability of the concavesurface type, so the cause of the streaky patterns remains at present a mystery.

Many other topics were considered at the meeting, but there is no space to describe them here. It will already be evident, however, how abundantly fruitful has proved the concept of the boundary layer, first thought of by Prandtl more than fifty years ago, in 1904. In the decade following that year scientific papers on boundary layers appeared at the rate of about one a year. The current rate has been estimated to be about 160 a year, and this rate is still increasing. Despite all this effort, we are still a long way from understanding many boundary-layer phenomena. The attempt to solve these problems is one of the most interesting tasks of present-day aerodynamics, a task which is likely to take at least another fifty years to complete.
G. E. GADD

\section{THE BRITISH LEATHER MANUFACTURERS' RESEARCH ASSOCIATION}

$\mathrm{T}$ WHE British Leather Manufacturers' Research Association held its fortieth annual open days during May 3-5 at Milton Park, Egham, the Surrey mansion where the laboratories of the Association have been established for the past ten years. More than four hundred visitors attended, mostly from the industry, but including representatives of university depart. ments, research associations, government departments and other bodies.

The Association, which was one of the earliest to be established under the Department of Seientific and Industrial Research scheme for co-operative research in industry, has an income of $£ 65,000$ p.a., and applies its effort in roughly equal proportions to background research, technological investigations and member services. Studies of the composition of skin have been maintained since Dr. Joane H. Bowes, the Association's chief biochemist, worked out the overall amino-acid composition of collagen some fifteen years ago; in this year's exhibition the decrease with increasing age of the animal in the proportions of serum and other non-collagenous proteins in the skin was shown. The Biochemistry Department is making a fundamental investigation (sponsored by the U.S. Department of Agriculture) on the mechanisms of the deterioration of leather by various agencies such as heat, moisture and perspiration. A survey of the rates of deterioration of different kinds of leather was shown, together with an exhibit of the more fundamental aspects of the work, which involve the use of continuous paper electrophoresis and liquid-phase chromatography, to identify the breakdown products of the protein fibres and the tanning agents, and the use of isotopic labelling, to follow the movement of tanning agents out of the protein phase.

In the Physics Department, under Dr. R. G. Mitton, work on waterproofing of leather was shown. While the intrinsic water-resistance of leather is adequate for many purposes, there are increasing demands for special leathers with improved performance. Results obtained with a variety of treatments designed to improve water-resistance without reducing the porosity of leather were shown. An important objective of the work of the Physics Department is to devise laboratory tests for assessing the complex physical properties of leather in its many uses; a number of test machines constructed 